\title{
Personen mit Führungs- und Managementqualitäten
}

\author{
Marie-Paule Fauchère ${ }^{a}$, Bruno Gutknecht ${ }^{b}$, Thomas Heuberger ${ }^{c}$ \\ a Präsidentin Association Romande des Assistantes Médicales ARAM \\ ${ }^{b}$ Fürsprecher, Geschäftsführer OdA Berufsbildung MPA und Zentralsekretär Schweizerischer Verband Medizinischer Praxisassistentinnen SVA \\ c Dr. med., Präsident OdA Berufsbildung MPA
}

Die Association Romande des Assistantes Médicales ARAM, die Hausärzte Schweiz, der Schweizerische Verband Medizinischer Berufsschulen svmb, der Schweizerische Verband Medizinischer Praxis-Assistentinnen SVA und die Verbindung der Schweizer Ärztinnen und Ärzte FMH haben gemeinsam eine Weiterbildungsstufe für Medizinische Praxisassistentinnen in der Form einer Berufsprüfung mit eidgenössischem Fachausweis erarbeitet. Nachdem der BSMPA seine Einsprache gegen die Publikation der Prüfungsordnung beim Staatssekretariat für Bildung, Forschung und Innovation SBFI zurückgezogen hat, ist die Prüfungsordnung am 5. Februar 2015 in Kraft getreten. Die erste Berufsprüfung findet Anfang Dezember 2015 statt. Für alle weiteren Informationen siehe www.odamed.ch.

\section{Berufsbilder}

Für die Medizinische Praxiskoordinatorin sind zwei Einsatzbereiche vorgesehen. Entsprechend dazu wird die Prüfung in zwei Fachrichtungen abgenommen.

\section{Medizinische Praxiskoordinatorin / Medizini- scher Praxiskoordinator klinischer Richtung} Medizinische Praxiskoordinatorinnen oder Medizinische Praxiskoordinatoren klinischer Richtung arbeiten in einer Arztpraxis und betreuen unter der Verantwortung einer Ärztin oder eines Arztes Menschen mit einer oder mehreren chronischen Krankheiten. Dabei erfassen sie die aktuelle Lage der Patientin oder des Patienten, planen gestützt auf evidenzbasierte Vorgaben die weitere Betreuung, fördern ihre oder seine Selbsthilfekompetenz und die Pflegekompetenz der Angehörigen, koordinieren Leistungserbringer und erleichtern den Zugang zu den kommunalen Ressourcen. Sie kennen die wichtigsten Aufgaben und Handlungskompetenzen der Fachpersonen im Gesundheitswesen und respektieren ihre eigenen fachlichen und beruflichen Grenzen.

Bei Krankenkassen und Versicherungen können sie als Gruppenleiterinnen oder Gruppenleiter im Rahmen des Case Managements zum Einsatz kommen.

\section{Medizinische Praxiskoordinatorin / Medizinischer Praxiskoordinator praxisleiten- der Richtung}

Medizinische Praxiskoordinatorinnen oder Medizinische Praxiskoordinatoren praxisleitender Richtung verbinden klinisch-medizinische Erfahrung mit Geschäftsführungs-Knowhow. Sie arbeiten in einer Kaderfunktion und sind in einer Arztpraxis mit dem selbständigen Führen des Personals und des Personalwesens, des Einkommensmanagements, des Praxismarketings und der Qualitätssicherung betraut. Bei Krankenkassen und Versicherungen können sie als Gruppenleiterinnen oder Gruppenleiter im Rahmen des Case Managements zum Einsatz kommen.

\section{Ein Bedürfnis}

Mit dem Erwerb des Fachausweises erhält die MPA erstmals die Gelegenheit, auch formell mit einem eidgenössisch anerkannten Abschluss aus der bildungssystematisch vorgegebenen und traditionellen subalternen Position aufzusteigen und sich dabei gleichzeitig neue Möglichkeiten der beruflichen Mobilität zu erschliessen. Eine Erweiterung der Kompetenzen der Praxiskoordinatorin im klinischen Fach zielt auf die selbständige Beratung von Langzeitpatienten in Bereichen, wo die ärztlichen Qualifikationen nicht zwingend gefordert sind. Damit wird der Einsatz der Praxiskoordinatorin klinischer Richtung bei Hausärztinnen und Hausärzten interessant. Die Verhandlungen mit Behörden und Versicherern mit dem Ziel, für MPK Beratungsleistungen abrechnen zu können, laufen.

Die Trägerschaft stellt in der zunehmenden Zahl von Gruppen-, Grosspraxen und Ärztenetzwerken ein steigendes Bedürfnis nach Leaderpersonen im Praxisteam fest. Gesucht sind Personen mit Führungs- und Managementqualitäten, die es den Praxisinhabern erlauben, sich von administrativen und organisatorischen Aufgaben zu entlasten, damit sie sich primär ihrer eigentlichen Berufsfunktion als Ärztin oder Arzt zuwenden können. 


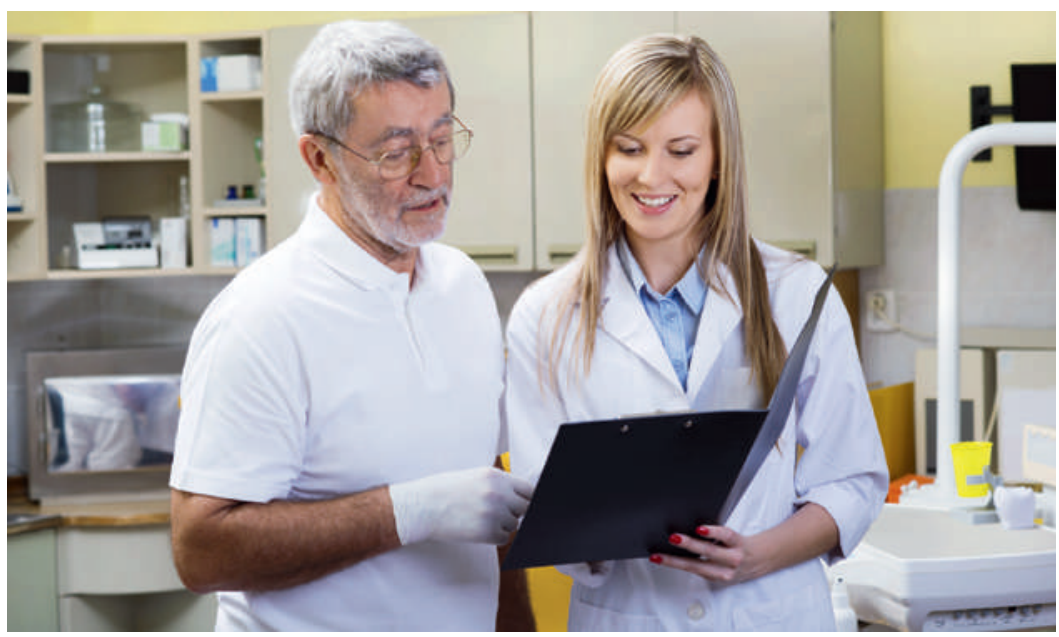

Die neuen Berufsbilder bieten die Möglichkeit, auch formell aus einer subalternen Position aufzusteigen.
Zur Abschlussprüfung in der praxisleitenden Richtung wird zugelassen, wer

- ein Eidgenössisches Fähigkeitszeugnis als Medizinische Praxisassistentin oder Medizinischer Praxisassistent, ein Eidgenössisches Fähigkeitszeugnis als gelernte Medizinische Praxisassistentin oder gelernter Medizinischer Praxisassistent, ein Arztgehilfinnen-Diplom DVSA besitzt oder über eine andere äquivalente Ausbildung der Sekundarstufe II oder der Tertiärstufe verfügt;

- über eine Berufspraxis von mindestens 3 Jahren nach Abschluss der beruflichen Grundbildung in allen Arbeiten der Arztpraxis oder einer vergleichbaren Arbeitsstelle verfügt;

Für beide Richtungen gilt als Zulassungsbedingung, dass die Kandidatin oder der Kandidat

- einen Kurs für Berufsbildnerinnen und Berufsbildner absolviert hat;

- über die erforderlichen Modulabschlüsse bzw. Gleichwertigkeitsbestätigungen verfügt.

\section{Module}

Folgende Modulabschlüsse resp. Gleichwertigkeitsbestätigungen müssen für die Zulassung zur Abschlussprüfung vorliegen:

\section{Klinische Richtung}

\section{Pflichtmodule}

- Chronic Care Management (Basismodul I)

- Chronic Care Management (Basismodul II)

- Qualitätsmanagement in der Arztpraxis

- Beratung von Langzeitpatienten - Diabetes

\section{Zulassung zur Berufsprüfung}

Zur Abschlussprüfung in der Klinischen Richtung wird zugelassen, wer

- ein Eidgenössisches Fähigkeitszeugnis als Medizinische Praxisassistentin oder Medizinischer Praxisassistent, ein Eidgenössisches Fähigkeitszeugnis als gelernte Medizinische Praxisassistentin oder gelernter Medizinischer Praxisassistent, ein Arztgehilfinnen-Diplom DVSA mit Röntgenberechtigung Thorax/Extremitäten besitzt oder über eine andere äquivalente Ausbildung der Sekundarstufe II oder der Tertiärstufe mit Röntgenberechtigung Thorax/ Extremitäten verfügt;

- über eine Berufspraxis von mindestens 3 Jahren nach Abschluss der beruflichen Grundbildung in allen Arbeiten der Arztpraxis gemäss Verordnung über die berufliche Grundbildung der Medizinischen Praxisassistentin / Medizinischer Praxisassistent verfügt;

\section{Zwei der folgenden Wahlpflichtmodule}

- Beratung von Langzeitpatienten - Rheuma

- Beratung von Langzeitpatienten - Atemwegserkrankungen

- Beratung von Langzeitpatienten - Koronare Herzkrankheit/Herzinsuffizienz

- Hirnleistungsschwäche

- Wundbehandlung

- Dosisintensives Röntgen

\section{Praxisleitende Richtung}

\section{Pflichtmodule}

- Chronic Care Management (Basismodul I)

- Chronic Care Management (Basismodul II)

- Qualitätsmanagement in der Arztpraxis

- Praxismanagement

- Personalführung 
Eines der folgenden Wahlpflichtmodule

- Rechnungswesen

- Vertiefte EDV-Kompetenzen für die Arztpraxis

- Wiederaufbereitung von Medizinprodukten

- Dosisintensives Röntgen

Alle Module werden in der Deutschschweiz von verschiedenen Bildungsanbietern angeboten, in der Westschweiz und im Tessin sind die Entwicklungsarbeiten im Gang. Wer bereits vor Inkrafttreten der Prüfungsordnung eines der von der OdA zum Modulbaukasten der Prüfung ausgewählten Module besucht hat, kann damit rechnen, dass sein Lehrgang an die Prüfung angerechnet wird. Damit gehen keine Bildungsleistungen verloren. Die Modulanbieter, Bildungsinstitutionen und Verbände müssen ihre Module und sich selbst als Modulanbieter bei der OdA zertifizieren lassen.

\section{Refresherkurse}

Da die Gültigkeitsdauer der Modulabschlüsse generell auf fünf Jahre limitiert ist, werden einzelne Modulanbieter für die Teilnehmerinnen an früheren Lehrgän- gen mit abgelaufenem Gültigkeitsdatum Refresherkurse anbieten, damit das Gültigkeitsdatum des Modulabschlusses für die Zulassung zur Berufsprüfung erneuert werden kann. Bei welchen Modulen der Besuch von Refresherkursen nach Ablauf der Gültigkeitsdauer überhaupt verlangt wird, entscheidet die OdA aufgrund der Tatsache, wie weit die Modulinhalte nach Ablauf der Fünfjahresfrist als veraltet gelten müssen (z.B. neue Forschungs- und Praxiserkenntnisse im Diabetes).

\section{Gleichwertigkeitsverfahren}

In der Bildungslandschaft rund um den Beruf der Medizinischen Praxisassistentin sind in den letzten Jahren viele Weiterbildungsgefässe angeboten worden, die inhaltlich ganz oder teilweise den definierten Modulen entsprechen. Vergleichbare Bildungsleistungen sollen grundsätzlich angerechnet werden können. Die Qualitätssicherungskommission wird in jedem Einzelfall in einem Gleichwertigkeitsverfahren überprüfen, ob eine Anrechnung stattfinden kann oder nicht.
Korrespondenz:

Dr. med. Thomas Heuberger

OdAmed

Monbijoustrasse 35

Postfach 6432

CH-3001 Bern

Tel. 0313805458

info[at]odamed.ch
Prüfungsexperten und Prüfungsexpertinnen für die Berufsprüfung Medizinische Praxiskoordinatorin und Medizinischer Praxiskoordinator (klinische und praxisleitende Richtung)

Am 3. Dezember 2015 finden die ersten Berufsprüfungen zur Medizinischen Praxiskoordinatorin / zum Medizinischen Praxiskoordinator mit Eidgenössischem Fachausweis statt. Für diesen neuen Weiterbildungsweg der Medizinischen Praxisassistentinnen auf Tertiärstufenebene suchen wir Prüfungsexpertinnen und -experten. Alle Informationen über die zuführenden Module und den Prüfungsanlass finden sich auf www.odamed.ch

Die Expertenaufgaben umfassen die Beurteilung schriftlicher Fallstudien, die Durchführung des Fachgesprächs zur Fallstudie und die Bewertung der schriftlichen Prüfung. Zum Anforderungsprofil: Sie

- verfügen über eine abgeschlossene Ausbildung als Ärztin oder Arzt und über eine mehrjährige Berufserfahrung in der ambulanten Grundversorgung, im Chronic Care Management und im Qualitätsmanagement in der Arztpraxis;

- besitzen pädagogische und methodisch-didaktische Fähigkeiten bzw. Erfahrung in der Berufsbildung;
- bringen Interesse und Engagement für die Entwicklung des Berufs der Medizinischen Praxisassistentin / des Medizinischen Praxisassistenten und der Weiterbildungsstufe mit. Alle Expertinnen und Experten besuchen vor ihrem Experteneinsatz einen eintägigen Ausbildungskurs für Prüfungsexpertinnen und -experten Niveau Höhere Berufsbildung beim Eidgenössischen Hochschulinstitut für Bildung (EHB).

Ebenfalls gesucht werden Mentorinnen und Mentoren, die die Kandidatinnen bei der Abfassung der schriftlichen Fallstudie bei Bedarf begleiten können.

Sowohl die Experten- wie die Mentorentätigkeit werden durch die Prüfungsträgerschaft im Rahmen der Richtlinien des Staatssekretariats für Bildung, Forschung und Innovation SBFI entschädigt.

Auskünfte erteilt die Geschäftsstelle der OdA Berufsbildung Medizinische Praxisassistentin odamed, Monbijoustrasse 35 Postfach 6432, 3001 Bern, Tel. 03138054 58, www.odamed.ch, info[at]odamed.ch 